\title{
EL SUEÑO DEL VOLUNTARIADO DEL GRUPO INTERACTIVO EN EDUCACIÓN FÍSICA
}

\author{
Francisco Javier Giles Girela \\ Carmen Trigueros Cervantes \\ Enrique Rivera García \\ Universidad de Granada
}

\begin{abstract}
RESUMEN: Este artículo tiene el objetivo de analizar las inquietudes y percepciones del alumnado universitario en una experiencia de AprendizajeServicio en Comunidades de Aprendizaje. El contexto de la investigación se sitúa en cuatro centros educativos situados en zonas de exclusión social, y 36 estudiantes de la asignatura de Enseñanza de la Educación Física del Grado de Educación Primaria. La producción de la información se ha realizado mediante estrategias cualitativas, concretamente a través de un cuestionario abierto, utilizando para su análisis una metodología mixta de análisis categorial apoyado por el NVivo. Los principales hallazgos de la investigación muestran que el alumnado universitario muestra una excelente predisposición hacia la participación en este tipo de propuestas, buscando en ellas el desarrollo de competencias generales y profesionales del título. Por último y en base a todo lo anterior, podríamos señalar que las propuestas de Aprendizaje-Servicio ofrecen una amplia gama de experiencias y conocimientos para el desarrollo profesional de los futuros docentes.
\end{abstract}

PALABRAS CLAVE: sueño del voluntario, aprendizaje servicio, comunidades de aprendizaje, grupos interactivos, educación física.

\section{THE INTERACTIVE GROUP VOLUNTEERING DREAM IN PHYSICAL EDUCATION}

\footnotetext{
ABSTRACT: The main objective of this report is to analyze the university students concerns and perceptions regarding their participation in a ServiceLearning experience in Learning Communities. The context research is located in four educational centers situated in exclusion areas and, in the Physical Education Teaching students of the Primary Education Degree of Physical Education Subject. The information was produced using qualitative strategies,
} 
specifically through an open questionnaire, using for its analysis a mixed strategy of categorical analysis supported by NVivo. The main research findings show that students have a maximum predisposition towards participation in this type of proposal to develop their skills and acquire personal and professional skills for their learning. Finally and based on all of the above, we could point out that the Service-Learning proposals offer a wide range of experiences and knowledge for the professional development of future teachers.

KEYWORDS: Volunteer dream, service learning, learning communities, interactive groups, physical education.

Recibido: 08/04/2020

Aceptado: 03/06/2020

Correspondencia: Francisco Javier Giles Girela, Facultad de Ciencias de la Actividad Física y del Deporte, Universidad de Granada, Camino de Alfacar, 21, 18071 Granada. Email: javiggr@hotmail.com

\section{INTRODUCCIÓN}

Desde hace tiempo, la Universidad Ileva intentando Ilevar a cabo metodologías que favorezcan la construcción de aprendizajes relevantes para el futuro desarrollo social y profesional del alumnado. Según Hargreaves y Fink (2008), esto depende directamente de la implementación de propuestas innovadoras que hagan salir de la zona de confort hacia un aprendizaje más autónomo del alumnado.

Por otro lado, y en relación con la asignatura de Educación Física, una de las claves para impulsar los cambios que precisa se encuentra en establecer un nuevo diálogo entre el alumnado y el aprendizaje a través de nuevas metodologías de enseñanza. En esta dirección, las Comunidades de Aprendizaje (CdA) y, más concretamente, el trabajo en el aula desde los grupos interactivos, se convierte en una estrategia excelente para superar las situaciones de desigualdad educativa generando igualdad de oportunidades de aprendizaje tal y como veremos a continuación.

Comenzando con las $\mathrm{CdA}$, podríamos señalar que estas se plantean como un cambio integral en la filosofía y organización de los centros que buscan cambios en la comunidad y la transformación de su entorno (Díez-Palomar y Flecha, 2010; Elboj, Puigdellivol, Soler y Valls, 2002; García, Leena y Petreñas, 2013). Su implementación conlleva el desarrollo de Actuaciones Educativas de Éxito (AEE), definidas en el proyecto de investigación INCLUD-ED, para llevar a cabo una transformación social y educativa (Díez-Palomar y Flecha, 2010).

Centrando nuestra atención hacia el trabajo desde los grupos interactivos (GI), estos se convierten en una estrategia metodológica y organizativa eficaz, que tiene como objetivo mejorar un aprendizaje determinado mediante la organización del aula en grupos heterogéneos (Ferrer, 2005). En cada grupo se realizará una actividad 
tutorizada por una persona adulta voluntaria (en nuestro caso, el alumnado universitario), facilitándose la organización del aula, la adquisición del aprendizaje y la mejora de las interacciones de los niños y niñas. Para Girbés (2011) y Molina (2007), el rol del voluntariado es generar ayuda mutua entre los componentes del grupo y fomentar las interacciones que aceleren el aprendizaje. Vemos, por tanto, que la actitud del voluntario es fundamental para brindar al alumnado la oportunidad de ser los auténticos protagonistas de su propio aprendizaje cumpliendo las siguientes funciones: asegurar que todos participen en la actividad con éxito en su resolución, promover ayuda y solidaridad entre iguales, ser partícipe en la evaluación para mejorar la propuesta y velar por el control y buen funcionamiento de la actividad, adquiriendo, por tanto, un compromiso con las necesidades humanas y comunitarias del entorno escolar. Por otro lado, el profesorado será el encargado de planificar la sesión, preparar las actividades para cada grupo, coordinar y observar resolviendo dudas y coordinándose con el voluntario. Su papel será el de organizar las actividades, estructurar toda la clase, llevar los tiempos y controlar que todos los grupos trabajen correctamente en colaboración con el voluntariado. Numerosos autores (Aubert, Medina y Sánchez, 2000; Molina, Robles y Sánchez, 2011; Píriz, 2011) respecto al alumnado que recibe la propuesta, coinciden en que tienen conocimiento previo sobre el funcionamiento y los beneficios que aportan los GI sobre su aprendizaje, participando en su evaluación. Además, estos autores muestran unanimidad en la actitud positiva del alumnado ante esta actuación, sintiéndose motivados hacia el aprendizaje. En los Gl, el alumnado de primaria crea conocimientos a través del diálogo, fomentando el desarrollo de valores personales en estos (Aubert et al., 2008; Díez-Palomar, García, Molina y Rué, 2010). Tras lo expuesto anteriormente, podríamos considerar que las CdA y la puesta en práctica del Grupo Interactivo Corporal en nuestras clases de Educación Física (Giles, Trigueros y Rivera, 2020), facilita la organización del aula, la adquisición del aprendizaje y la convivencia entre el alumnado, convirtiéndose en una herramienta excelente para superar las situaciones de desigualdad educativa y generar oportunidades de aprendizaje para todo el alumnado participante en la propuesta.

Desde la óptica del Aprendizaje-Servicio Universitario (ApSU) y la presencia del alumnado universitario en el aula de Educación Física como voluntario-a en los Grupos Interactivos Corporales de las CdA, decir que se convierte en una excelente estrategia para nutrir a los centros de voluntarias y voluntarios que faciliten la puesta en práctica de los principios del aprendizaje dialógico, creados a partir de las aportaciones de autores como Vygotsky, Bruner, Wells, Paulo Freire, Habermas, Chomsky, Scribner y Mead (Aubert et al., 2008). Además, el ApSU se convierte aquí en una metodología clave para el desarrollo de competencias clave mientras el alumnado ofrece un servicio a la comunidad (Moliner, Francisco y Gil, 2010; Torío, Peña y Hernández, 2014). Entendiendo al ApSU como una metodología que insiste en el aprendizaje académico a través de prestar un servicio a colectivos vulnerables, el análisis de los puntos fuertes y débiles percibidos por los estudiantes debería ser un aspecto fundamental para mejorar el diseño de futuras propuestas (Gil, 2012). Las experiencias proporcionadas al alumnado a través de esta vía, les ofrece la oportunidad para poner en funcionamiento recursos que habitualmente no pone en práctica 
en las clases teóricas o en las simulaciones prácticas realizadas en la Facultad (Gil, Chiva y Martí, 2013). Además, promueve valores como: superación personal ante las dificultades de comprensión, análisis, síntesis, crítica, seriedad, rigor y duda, autocrítica y tesón (Campo, 2014), propiciando escenarios excelentes para el desarrollo de la ética, la creatividad, el análisis y el esfuerzo personal. Añadiendo a ello el desarrollo de habilidades sociales y cooperativas como diálogo, escucha activa, empatía y compromiso, remarcando el ApS como una metodología clave para aprender a hacer, aprender a ser, aprender a vivir y saber gestionar las interacciones sociales que se den en el aula (Francisco y Moliner, 2010). Mendía (2012) señala que el ApSU:

...promueve valores... Facilita experiencias de confianza interpersonal, desarrolla la capacidad asociativa, fomenta la conciencia cívica y los valores de la ética, estimula el emprendimiento social, el aprendizaje de habilidades o destrezas que ayudan a las personas a enfrentarse adecuadamente a las exigencias y desafíos de la vida diaria (pp. 73-74).

En este sentido y refiriéndonos al desarrollo de competencias para el alumnado universitario que realiza ApS, este ofrece el desarrollo de: trabajo en equipo, interdisciplinariedad, mejora de las relaciones personales, compromiso ético, razonamiento crítico, entre otros; mientras compensa a la comunidad en base a sus demandas (Moliner, Amat y Gil, 2010). Desde una perspectiva instrumental, siguiendo a Alonso, Arandia, Martínez, Martínez y Gezuraga, (2013), el principal objetivo de la puesta en práctica de estas propuestas se centra en la capacidad del alumnado para dinamizar, organizar y gestionar el grupo y proyecto de ApSU, utilizando las técnicas y estrategias más adecuadas para la resolución de problemas y dificultades encontradas durante el mismo. Con relación al desarrollo de competencias centradas en el saber ser y estar, numerosos autores (Furco y Billig, 2002; Hernández, Larrauri y Mendía, 2009; Tapia, 2008; Wilczenski y Cook, 2009), indican que el ApSU es ideal para trabajar las competencias emocionales. Completando la idea anterior, autoras como Lorenzo y Matallanes (2013), ven en el ApS un enorme potencial para desarrollar competencias sociales y emocionales en el alumnado universitario. En este sentido, Chiva, Ruiz, Martín, Pérez, Giles, García y Rivera (2019) ponen de manifiesto la necesaria reorientación de la praxis que eduque en competencias, remarcando el enorme potencial del ApSU para promover competencias sociales y personales.

Ante lo expuesto, nuestra pregunta de investigación la hemos querido centrar en el antes, frente al después y, en este sentido, nos ha interesado especialmente conocer si los sueños previos de nuestro alumnado participante en el ApSU vinculado a las Comunidades de Aprendizaje, quedaban reflejados en las competencias que otras investigaciones han hecho emerger desde la evaluación final.

Nos ha parecido clave indagar sobre las motivaciones que les ha llevado a elegir esta opción formativa frente a las propuestas habituales. Hasta dónde entienden que puede llegar su compromiso con el ApSU y qué pueden aportar desde su experiencia previa. Por último, intentaremos conocer y analizar las expectativas que tienen en cuanto a las competencias que pueden adquirir desde la experiencia vivida. 


\section{Método}

Esta propuesta se ha llevado a cabo dentro de un programa de ApSU puesto en práctica en la asignatura de Enseñanza de la Educación Física de la Facultad de Ciencias de la Educación de la Universidad de Granada, asistiendo a diferentes centros educativos constituidos como CdA y en colaboración con el docente del centro de Educación Física para el control y buen funcionamiento de los grupos interactivos propuestos.

En esta investigación ha existido un ApSU equilibrado en el que tanto el alumnado del centro como el docente han salido beneficiados del proceso por igual, existiendo un equilibrio entre el aprendizaje realizado y el servicio que el alumnado ha prestado en el proyecto. El alumnado universitario ponía en práctica lo aprendido en clase mientras el colegio llevaba a cabo la propuesta de grupos interactivos en Educación Física, mediante la ayuda del voluntariado mejorando aspectos como la escucha y respeto en el alumnado de primaria.

A partir del paradigma interpretativo con enfoque fenomenológico, teniendo en cuenta el objetivo principal de esta investigación, centramos nuestro análisis cualitativo en analizar las motivaciones del alumnado al tener la posibilidad de participar en un proyecto de ApS.

\subsection{Contextualización}

Habitualmente, el voluntariado que participa en las CdA está formado por familiares, personas del barrio, exalumnos y estudiantes universitarios, siendo este último colectivo el que compone nuestro proyecto.

Nuestro estudio se ha desarrollado con 36 estudiantes de $2^{\circ}$ Grado de Educación Primaria de la asignatura de Enseñanza de la Educación Física, participando como voluntarios en la propuesta. La implicación de las mujeres en la propuesta ha sido muy superior a la de los hombres: un $72,2 \%$ de alumnas frente a un $27,8 \%$ de alumnos. Los receptores del servicio han sido un total de cuatro centros educativos (Tabla 1) constituidos como CdA ubicados en la zona norte de Granada (barrio de Almanjayar); siendo un área de atención preferente, donde la mayoría del alumnado es de etnia gitana y todos ellos se encuentran en contextos de exclusión social de mayor o menor intensidad.

Tabla 1. Participantes en el proyecto. Fuente: elaboración propia

\begin{tabular}{|c|c|c|c|c|c|}
\hline \multicolumn{2}{|c|}{$\begin{array}{c}\text { Alumnado universidad } \\
\text { (36) }\end{array}$} & \multicolumn{4}{|c|}{$\begin{array}{c}\text { Receptores del servicio } \\
\text { (200) }\end{array}$} \\
\hline Mujeres & Hombres & Centro 1 & Centro 2 & Centro 3 & Centro 4 \\
\hline $72 \%$ & $28 \%$ & $23,00 \%$ & $27,00 \%$ & $24 \%$ & $26,00 \%$ \\
\hline
\end{tabular}

Podemos apreciar en la figura 1, los tres grandes momentos por los que pasa la propuesta que se realiza de Aprendizaje-Servicio Universitario vinculado a los cen- 
tros de Comunidades de Aprendizaje. Partimos de un tiempo de formación, realizado fuera del horario lectivo en 8 horas de taller. A partir de esta formación, se acude al centro para colaborar con el docente de Educación Física en las clases y muy especialmente en lo que venimos identificando como Pedagogía Dialógica Corporal. Estamos en la implementación del proyecto que conlleva simultáneos momentos de reflexión y evaluación grupal. Por último, en la fase tres, se realiza un espacio de reflexión y análisis de la experiencia mediante tutorías grupales por centros.

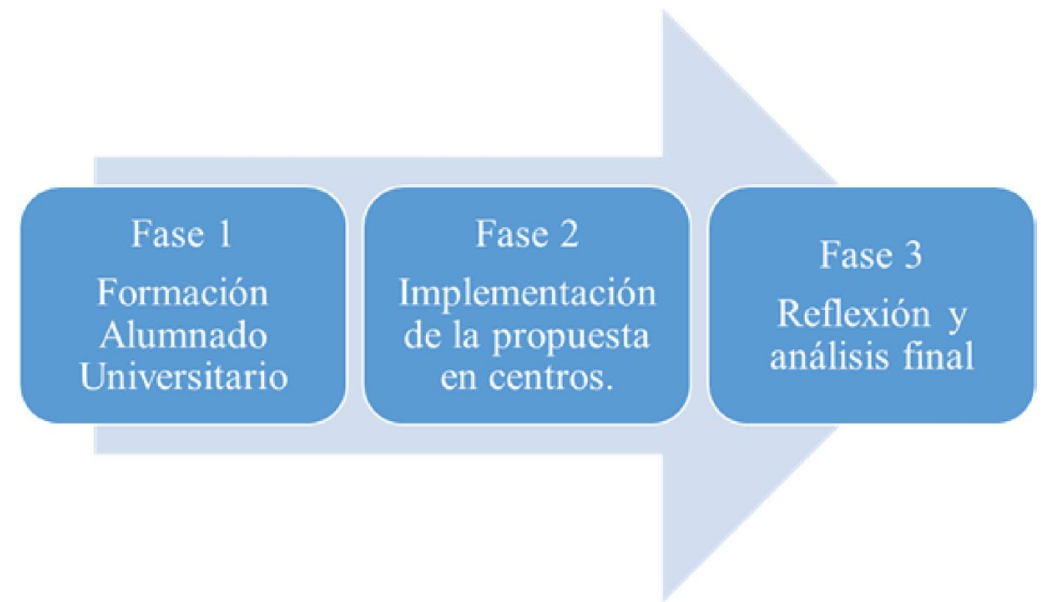

Figura 1. Fases contempladas en el proyecto. Fuente: elaboración propia

\subsection{Técnica de producción de la información}

La producción de la información se ha realizado a través de técnicas cualitativas, concretamente con un formulario abierto de Google Drive pasado al alumnado participante al inicio del proceso. En él, se le solicitaba que realizara una reflexión crítica acerca de sus inquietudes ante el proyecto dando respuesta a los interrogantes de: motivaciones para realizar el ApSU y perspectivas de dedicación; experiencia previa similar o en línea con trabajo de voluntariado, participación en escuelas deportivas, además de las competencias que considera que posee y le pueden facilitar la realización del ApSU propuesto. Hemos tenido en la clase un grupo que ha llevado una formación con metodología más tradicional y otro que sus prácticas se han desarrollado desde el ApSU.

Respecto a la estructura del cuestionario: el alumnado universitario y antes de realizar la propuesta de ApSU contestó a las siguientes preguntas: motivos para hacer ApS, Nivel de esfuerzo ApS, qué puedo aportar, qué competencias tengo y qué puedo recibir.

\subsection{Procedimiento de análisis}

Nuestro análisis ha seguido la propuesta planteada por Trigueros, Rivera y Rivera (2017, 2019) y Trigueros, Rivera, Moreno y Muñoz (2016). Se ha realizado un análisis categorial, partiendo de los interrogantes planteados en el cuestionario abierto, en 
combinación con una categorización inductiva. Para su realización se ha contado con la ayuda del software NVivo.

El punto de partida inductivo se ha concretado en una identificación de los conceptos clave mediante una frecuencia de palabras de las narrativas aportadas por el alumnado. Como podemos ver en la figura 2, se establecieron cinco grandes categorías coincidentes con los interrogantes mencionados anteriormente y que se identifican como: motivaciones previas, nivel de esfuerzo y compromiso, competencias que identifica en su persona para afrontar el reto del ApSU, qué piensa que puede aportar al proceso y qué perspectivas de aprendizaje tiene una vez finalizado el ApSU.

\begin{tabular}{|c|c|c|c|c|}
\hline (๑) & Name & , $\leftrightarrow$ & Files & References \\
\hline$\square 0$ & Motivos para hacer APS & & 1 & 36 \\
\hline & Aprendizaje & & 1 & 6 \\
\hline & Docencia & & 1 & 7 \\
\hline & Experiencia & & 1 & 14 \\
\hline & Oportunidad & & 1 & 6 \\
\hline-0 & Nivel de esfuerzo ApS & & 1 & 36 \\
\hline & ○ Máximo & & 1 & 13 \\
\hline & O Medio & & 1 & 11 \\
\hline-0 & Qué competencias tengo & & 1 & 36 \\
\hline & Ayuda & & 1 & 4 \\
\hline & Con. Activ. & & 1 & 7 \\
\hline & Conocimientos & & 1 & 3 \\
\hline & Creatividad & & 1 & 4 \\
\hline & O Empatía & & 1 & 3 \\
\hline & Paciencia & & 1 & 6 \\
\hline$\square 0$ & Qué puedo aportar & & 1 & 36 \\
\hline & Cursos & & 1 & 5 \\
\hline & Exp. niños & & 1 & 25 \\
\hline-0 & Qué puedo recibir & & 1 & 36 \\
\hline & Actividades & & 1 & 7 \\
\hline & Aprendizaje & & 1 & 9 \\
\hline & O Metodología & & 1 & 8 \\
\hline
\end{tabular}

Figura 2. Categorías analizadas. Fuente: elaboración propia 
El análisis parte de 180 referencias aportadas por el alumnado sobre los grandes temas identificados. Una vez identificados desde la frecuencia de palabras los conceptos más reiterados en los discursos, nos quedamos con los 50 más significativos que fueron categorizados en cada una de las cinco categorías identificadas. La recategorización de cada una de ellas nos llevó a identificar las subcategorías que se muestran en la figura 2; quedando constituida la codificación axial que nos servirá de punto de partida para realizar el posterior análisis e interpretación de los resultados.

\section{ANÁLISIS E INTERPRETACIÓN DE LOS RESULTADOS}

Iniciamos el análisis de los resultados visualizando la presencia o ausencia de cada una de las subcategorías que se han concretado en las cinco grandes categorías de partida. Este análisis es meramente cuantitativo, basado en las referencias anidadas en cada una de las subcategorías, por lo que no deja de ser una aproximación a la interpretación del qué, cómo y porqué expresan a nivel discursivo y el peso otorgado por los participantes al mismo.

Podemos visualizar (Figura 3) el primer sueño del alumnado: aportar su experiencia en el trato con los niños y niñas de primaria, en aquellos casos del alumnado que ha tenido o tienen la oportunidad de haber trabajado en situaciones de práctica real o adquirirla, y en aquellos otros casos que no han vivido aún la oportunidad de trabajar con niñas y niños de estas edades. Esta adquisición o ampliación de experiencia, coincide con lo planteado por Mendía (2012), cuando hace referencia a que una de las principales motivaciones del alumnado universitario para acercarse al ApSU es precisamente tener la oportunidad de ganar experiencia en su formación como docentes.

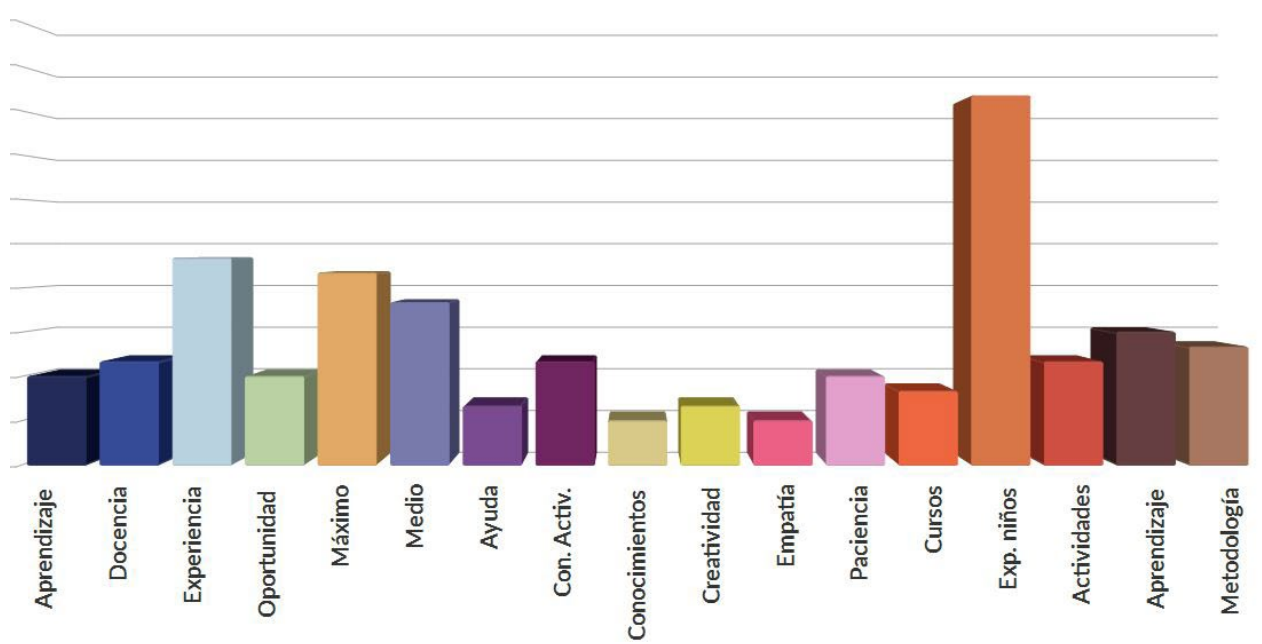

Figura 3. Consulta de codificación de subcategorías. Fuente: datos de la investigación 
En esta misma dirección se encaminan las palabras de María, cuando expresa que se trata de una "...oportunidad para ganar experiencia con niños y niñas y mejorar [su] formación" siendo "...muy útil conocer diferentes metodologías para el día de mañana...enfrentarse a próximos alumnos". Esta segunda reflexión, se enmarca perfectamente por lo planteado por Hargreaves y Fink (2008) cuando hacen referencia a la necesidad de comenzar a implementar en el aula en general y en la universitaria en particular, metodologías disruptivas que rompan las actuales inercias formativas y logren motivar al alumnado hacia el aprendizaje frente al simple logro de una calificación positiva en la materia. Patricia ve al ApSU como "...una oportunidad para aprender de otros profesionales de la docencia, para sentirme útil y aportar mis conocimientos"; o, como nos dice Anabel, sintiendo que la experiencia vivida es "...muy interesante poder disfrutar de esta experiencia innovadora en el campo de la educación". Ella misma, hace referencia a la importancia de "... tener una formación permanente..." en la que "...la escuela debe transmitir conocimientos significativos para el alumnado y teniendo en cuenta la competencia social y cívica". Carmen nos expresa que para ella es clave para su formación "...la motivación, el trabajo en grupo, participación, el contacto directo con el entorno, los debates y la creatividad..." en la que "...el aprendizaje no solo será de los alumnos/as...". Estas mismas ideas que aquí ha reflejado nuestro alumnado, ya han sido recogidas por autores como Moliner, Francisco y Gil (2010) o Torío et al. (2014). No solo la construcción de competencias instrumentales debe ser el principal referente de la formación docente; es clave aportar un bagaje axiológico y social que permee las competencias específicas, dotándolas de sentido en un contexto social donde la libertad y la solidaridad se conformen en los ejes que posibiliten una aproximación hacia la igualdad lo más real y posible de la ciudadanía. En esta línea, tanto las Comunidades de Aprendizaje como el ApSU pueden ser dos catalizadores fundamentales para mejorar una formación docente que valore el saber conceptual e instrumental, pero sustentado desde el crecimiento de la persona como integrante social. Carlos lo vive como una oportunidad para "...aprender a desarrollar [sus] habilidades docentes de una manera práctica e interactiva..." considerando que "...un aprendizaje basado en servicios puede ayudarme a completar mi formación". Como podemos ver, la luz se vislumbra tenuemente al final del túnel, pero debemos tomar conciencia de que una sola experiencia de ApS en una materia que supone poco más del dos por ciento de la formación, no deja de ser una leve apertura en el muro.

¿Cuánto tiempo puedo y quiero dedicar a la experiencia? Algunas estudiantes como Carmen quieren dedicar el máximo que puedan, ya que ven la posibilidad de realizar ApSU en Comunidades como "...una oportunidad única que nos brindan desde la asignatura de Educación Física", en la que la forma de proceder les parece "...una vía extraordinaria para un aprendizaje más profundo y real". Perciben que están ante una oportunidad única, no experimentada aún en su formación como docentes que, además, les brinda la oportunidad de poner en el empeño todas "...las ganas e ilusión por conocer esta metodología innovadora que considero fundamental en la vida diaria de nuestros futuros alumnos, padres y comunidades de aprendizaje en general". Como nos mencionaba Laura, es acercarse no solo a una metodología 
diferente, es tener la posibilidad de dar, recibir y devolver a toda la comunidad educativa. Porque, como dice Carlos, comprometerse significa tener la oportunidad de recibir, fundamentalmente, experiencia "...para poder afrontar cualquier obstáculo que nos encontremos en la docencia".

La tercera parada que hacen en su camino de voluntariado gracias al ApSU en centros de $\mathrm{CdA}$, lo sitúan en su formación como personas que desean ejercer en un futuro la docencia. Inma quiere "...poder ser útil a la sociedad, aportar un granito de arena e intentar mejorarla, sensibilizándome y comprometiéndome con las necesidades sociales reales...", al mismo tiempo que aprende "...distintas habilidades para desarrollar mi futura labor docente". Con sabiduría entreteje los dos saberes que la pueden formar como una excelente profesional, el conceptual e instrumental, con el de crecimiento personal y social. Esta idea la veíamos reflejada en otras investigaciones como la realizada por Moliner, Amat y Gil (2010). Anabel nos muestra el lado axiológico de la docencia cuando nos comenta que "...siempre me ha gustado a ayudar a los demás" considerándose "...una persona empática, responsable y con paciencia". La otra cara de la moneda nos la ofrece Inma cuando hace hincapié en la necesidad de tener "...habilidades organizativas, narrativas, observadoras y conocimientos sobre actividades", en la línea de lo que expresan Alonso et al. (2013), sobre la adquisición de habilidades desde el ApSU.

La creatividad es otro de los aspectos más referenciado en el discurso de los participantes. Consideran que la posibilidad de realizar un buen ApSU pasa, como nos comenta Carmen, por tener la "virtud de la creatividad". Además, en la línea que marcan Francisco y Moliner (2010), consideran la empatía y la paciencia como otras virtudes necesarias para tener éxito en la experiencia, aunque su planteamiento se proyecta más allá de la misma y las tendríamos que considerar como virtudes de un buen docente.

Soñar es fácil, lo complejo es hacer realidad el sueño. Desde esta premisa nos tenemos que preguntar ¿qué puede aportar el alumnado que decide participar en la propuesta? La mayoría del alumnado hace referencia a su formación en cursos y aprendizajes; en la misma línea que Silvia cuando nos expresa que se siente preparada para "...crear una clase, [aportar] los recursos de los que disponemos, saber motivar a los niños, hacer que se diviertan con el deporte". Otros como Carlos hacen referencia al aprendizaje adquirido en experiencias previas y la posibilidad de "...aplicarlo a sesiones de Educación Física en algunos aspectos". Clotilde señala que ha hecho "...voluntariado en centros de acogida de niños con problemas de 'inmersión social'", al igual que Lidia, que ha participado "...en programas de convivencia e integración con niños en riesgo de exclusión". Inma, por su parte, nos remarca la importancia de tener experiencia para "...ayudar en las tareas de cuidado de niños y niñas, organización y realización de actividades, animación, ayuda de monitores...". Como podemos ver, hay una gran sintonía con lo ya expresado por autores como Furco y Billig, (2002); Hernández, Larrauri y Mendía, (2009); Tapia, (2008); Wilczenski y Cook, (2009), que identifican el ApSU como un espacio de formación integral, en el que se buscará la creación, 
tanto de competencias instrumentales como personales y sociales. Pero no pensemos que todo el alumnado que opta por el ApSU tiene experiencias previas; también hay un número significativo de ellos que van a recibir su bautismo de experiencia real, como le ocurre a Isabel cuando nos dice que "...nunca he trabajado con niños".

Pero los sueños se nutren de las expectativas que ponemos en ellos y lo más importante para el alumnado no se sitúa sólo en el dar, también el recibir es algo que demandan después del esfuerzo realizado. Partiendo de la definición de ApS que da Gil (2012), en la que remarca la gran oportunidad para el alumnado de aplicar el aprendizaje mientras realiza un servicio a la comunidad, encontramos a Alba que se siente motivada para "...llevar a la práctica los conocimientos adquiridos y poner en práctica otros nuevos como aprendizaje personal". Nos está diciendo que quiere dar, pero también recibir experiencia. Patricia, situándose en un plano de mayor concreción percibe la "...oportunidad para ver cómo actúan los niños en determinadas situaciones de aprendizaje". Lidia demanda el poder visualizar y experimentar "... un aprendizaje distinto al tradicional que considero útil y necesario en la formación", confirmando lo que propone Gil et al. (2013). Otras, como Natalia, quieren motivación y esperan poder encontrarla en las actividades propuestas indicando que "...son un aspecto que motivan al alumnado ya que llaman la atención porque no son las típicas". Esto nos confirma lo expuesto por Ferrer (2005), cuando hace referencia al potencial de los Grupos Interactivos como metodología de trabajo y medio para transformar y cambiar el entorno, superando así las desigualdades con respecto al aprendizaje (Ferrer, 2005; Díez-Palomar y Flecha, 2010; Elboj et al., 2002; García, Leena y Petreñas, 2013). Anabel abre su esperanza en tener la oportunidad de conocer una metodología que "...enseña a los niños y niñas a trabajar en grupos cooperativos y a vivir en sociedad". Carmen nos resume perfectamente lo expuesto cuando nos dice que percibe y espera del ApSU y el trabajo en las Comunidades de Aprendizaje una

...metodología [que] me va a hacer ver que es muy importante la manera que utilizamos para enseñar a nuestros niños/as y que muchas veces obviamos lo que es la motivación, el trabajo en grupo, participación, el contacto directo con el entorno, los debates y la creatividad.

Pero si hay un sueño valioso y anhelado por el alumnado que participa en esta experiencia, es aquel que les sitúa en la emoción que significa, como nos comenta Coltilde de tener la posibilidad de recibir amor, "...mi mayor motivación es el cariño y apego que voy a recibir", resaltando todos los valores que remarca Mendía (2012). Estamos ante la posibilidad de cerrar el círculo de la teoría del Don: dar, recibir, devolver (Godbout, 2007); porque la posibilidad de recibir debe estar precedida del haber tenido la capacidad de dar al otro y, desde la recepción estaremos en disposición de devolver en base a lo recibido. 
Tabla 2. Presencia de las Subcategorías por atributos de los participantes. Fuente: elaboración propia

\begin{tabular}{|c|c|c|c|c|c|c|c|c|}
\hline \multirow[b]{2}{*}{ Subcategorías } & \multicolumn{4}{|c|}{ Futura Mención de Especialidad } & \multicolumn{2}{|c|}{ Sexo } & \multicolumn{2}{|c|}{ Situación laboral } \\
\hline & $\begin{array}{l}\text { Ed. } \\
\text { Físc. }\end{array}$ & Primaria & $\begin{array}{l}\text { Idio- } \\
\text { mas }\end{array}$ & $\begin{array}{c}\text { Músi- } \\
\text { ca }\end{array}$ & Mujer & Hombre & $\begin{array}{c}\text { Traba- } \\
\text { ja }\end{array}$ & $\begin{array}{c}\text { No Tra- } \\
\text { baja }\end{array}$ \\
\hline \multicolumn{9}{|l|}{ Aprendizaje } \\
\hline \multicolumn{9}{|l|}{ Docencia } \\
\hline \multicolumn{9}{|l|}{ Experiencia } \\
\hline \multicolumn{9}{|l|}{ Oportunidad } \\
\hline \multicolumn{9}{|l|}{ Máximo } \\
\hline \multicolumn{9}{|l|}{ Medio } \\
\hline \multicolumn{9}{|l|}{ Ayuda } \\
\hline \multicolumn{9}{|l|}{ Con. Activ. } \\
\hline \multicolumn{9}{|l|}{ Conocimientos } \\
\hline \multicolumn{9}{|l|}{ Creatividad } \\
\hline \multicolumn{9}{|l|}{ Empatía } \\
\hline \multicolumn{9}{|l|}{ Paciencia } \\
\hline \multicolumn{9}{|l|}{ Cursos } \\
\hline \multicolumn{9}{|l|}{ Exp. niños } \\
\hline \multicolumn{9}{|l|}{ Actividades } \\
\hline \multicolumn{9}{|l|}{ Aprendizaje } \\
\hline Metodología & & & & & & & & \\
\hline
\end{tabular}

El cierre del análisis lo vamos a realizar a partir de la visualización de los acuerdos y desacuerdos encontrados en base a los atributos de nuestros participantes. Se ha trabajado fundamentalmente con tres atributos: la futura mención que piensan estudiar en el último curso, el sexo y su situación laboral. En base a ellos, y desde el cruce de la información en la matriz que se muestra en la Tabla 1, podemos ver que no se producen grandes desacuerdos, visualizándose en la intensidad de cada uno de los valores de los respectivos atributos una cierta uniformidad. Nos viene a confirmar lo anteriormente expuesto cuando mencionábamos que uno de sus principales sueños era tener la oportunidad de ganar experiencia, poniendo en valor sus aportaciones previas en esta dirección.

Si es interesante ver las diferencias que se producen entre el alumnado con la mirada puesta en estudiar una mención específica, especialmente la de Educación Física, frente a aquellos que su deseo es ser maestras o maestros generalistas. Esta misma percepción la podemos ver en las diferencias que se producen por sexo o la situación laboral. Estamos, en definitiva, ante formas diferentes de ver una misma experiencia de aprendizaje. Por una parte, aparecen los sueños de aquellas y aquellos más apegados a los conocimientos instrumentales, como van a ser los que desean 
especializarse o están en una situación laboral que les hace ver la realidad de la enseñanza, la mayoría vinculados a actividades complementarias y de apoyo escolar. En el otro extremo del segmento se sitúan los que piensan que lo instrumental es valioso; pero, junto a ello, también cabe la formación de una persona que tendrá que saber desenvolverse en un contexto social.

\section{Conclusiones}

Los sueños tienen la ventaja de que siempre se sitúan en el futuro; pero, de alguna manera, vienen condicionados por el pasado y pueden condicionar el presente por vivir. El alumnado participante en esta experiencia nos ha dejado la percepción de que, en su paso por el $\mathrm{ApSU}$ en centros de $\mathrm{CdA}$, desean, en primer lugar, vivir una experiencia formativa diferente al modelo imperante.

Entienden que la metodología disruptiva propuesta les abrirá las puertas a las dos claves del proceso de aprendizaje: la motivación y aprender desde la realidad de la práctica. Dos factores que difícilmente suelen percibir en la formación excesivamente teórica que se ofrece en el aula universitaria.

Anhelan poder aportar su experiencia, en algunos casos teórica, construida desde su formación no universitaria, en la que han vivenciado aquello que desean mantener y lo que no dudarán en olvidar y no reproducir. Otros, con experiencia acumulada en contextos diversos, también quieren aportar su corto bagaje de conocimientos a la nueva experiencia por vivir.

Por último, sueñan con poder aproximarse al aprendizaje desde una doble mirada. Por una parte, necesitan adquirir herramientas docentes que, desde su instrumentalidad, les dote de recursos didácticos lo suficientemente sólidos como para atender a los niños y niñas. Pero también entienden que la instrumentalidad precisa de un acompañamiento axiológico que les conecte con la sociedad y los futuros ciudadanos y ciudadanas, que tendrán que dar solución a los retos globales a los que nos enfrentamos.

El Aprendizaje-Servicio y las CdA también precisan de un docente universitario comprometido, que entienda que la recuperación de la actitud crítica es básica para cambiar un modelo de investigación que se comprometa con la transformación social, abandonando el actual modelo meritocrático en el que nos vemos inmersos.

\section{REFERENCIAS BIBLIOGRÁFICAS}

Alonso, I., Arandia, M., Martínez, I., Martínez, B. y Gezuraga, M. (2013). El aprendizaje-servicio en la innovación universitaria. Una experiencia realizada en la formación de educadoras y educadores sociales. Revista internacional de educación para la justicia social (RIEJS), 2(2), 195-216.

Aubert, A., Flecha, A., García, C., Flecha, R. y Racionero, S. (2008). Aprendizaje dialógico en la sociedad de la información. Barcelona: Hipatia Editorial. 
Aubert, A., Medina, A. y Sánchez, M. (2000). De las agrupaciones flexibles a los grupos interactivos. VIII Conferencia de sociología de la educación. Barcelona: Centre de Recerca Social i Educativa.

Campo Cano, L. (2014). Aprendizaje servicio y educación superior. Una rúbrica para evaluar la calidad de proyectos. (Tesis doctoral, Universidad de Barcelona, España). Recuperado de http://www.tdx.cat/handle/10803/277560

Chiva, O., Ruiz, P., Martín R., Pérez, I., Giles, J., García, J. y Rivera, E. (2019). University Service-Learning in Physical Education and Sport Sciences: A systematic review. Revista Complutense de Educación, 30(4), 1147-1164.

Díez-Palomar, J. y Flecha, R. (2010). Comunidades de Aprendizaje: un proyecto de transformación social y educativa. Revista Interuniversitaria de Formación del Profesorado, 67(24,1), 19-30.

Díez-Palomar, J., García, P., Molina, S. y Rué, L. (2010). Aprendizaje dialógico en las matemáticas y en las ciencias. Revista Interuniversitaria de Formación del Profesorado, 67(24,1), 75-88.

Elboj, C., Puigdellivol, I., Soler, M. y Valls, R. (2002). Comunidades de Aprendizaje. Transformar la educación. Barcelona: Graó.

Ferrer Esteban, G. (2005). Hacia la excelencia educativa en las comunidades de aprendizaje. Educar, (35), 61-70.

Francisco, A. y Moliner, L. (2010). El Aprendizaje Servicio en la Universidad: una estrategia en la formación de ciudadanía crítica. REIFOP, 13(4).

Furco, A. y Billig, S. H. (2002). Service-Learning: The Essence of the Pedagogy. Advances in Service-Learning Research. Greenwich, CT: Information Age Publishing.

García, C., Leena, A. y Petreñas, C. (2013). Comunidades de Aprendizaje. Scripta Nova, 427(7). Recuperado de http://www.ub.edu/geocrit/sn/sn-427/sn427-7.htm

Gil, J. (2012). El aprendizaje servicio en la enseñanza superior: una aplicación en el ámbito de la educación física (Tesis doctoral, Universidad Jaume I, Castellón, España). Recuperado de http://www.tdx.cat/handle/10803/86937

Gil, J., Chiva, O. y Martí, M. (2013). La adquisición de la competencia social y ciudadana en la universidad mediante el Aprendizaje-Servicio: Un estudio cuantitativo y cualitativo en el ámbito de la Educación Física. Revista Internacional de Educación para la Justicia Social, 2(2), 89-108.

Giles, F. J., Trigueros, C. y Rivera, E. (2020). Cuerpo y motricidad. La pedagogía dialógica corporal. Tándem. Didáctica de la Educación Física, 67, 7-13.

Girbés, S. (2011). Los grupos interactivos: hacia el éxito de todos y todas. Periódico Escuela, suplemento Comunidades de Aprendizaje, 1, 1-2.

Godbout, J. (2007), Ce qui circule entre nous. Donner, recevoir, rendre. París: Seuil. 
Hargreaves, A. y Fink, D. (2008). El liderazgo sostenible. Siete principios para el liderazgo en centros educativos innovadores. Madrid: Morata.

Hernández, C., Larrauri, J y Mendía, R. (2009). Guía zerbikas 2: Aprendizaje y servicio solidario y desarrollo de las competencias básicas. Bilbao: Fundación Zerbikas.

INCLUD-ED Consortium (Coord.). (2011). Actuaciones de éxito en las escuelas europeas. Ministerio de Educación.

Lorenzo, V. y Matallanes, B. (2013). Desarrollo y evaluación de competencias psicosociales en estudiantes universitarios a través de un programa de aprendizaje servicio. Revista internacional de educación para la justicia social, 2(2), 155-176.

Molina, S. (2007). Los grupos interactivos: una práctica de las comunidades de aprendizaje para la inclusión del alumnado con discapacidad. (Tesis doctoral). Universidad de Barcelona. Barcelona.

Molina. F., Robles I. y Sánchez C. (2011). Nueva metodología para dar respuesta a la diversidad del aula: grupos interactivos. En J. Navarro (Coord.), Diversidad, Calidad y Equidad Educativas. Murcia: Consejería de Educación, Formación y Empleo. Experiencia. Recuperado de. http://eoepsabi.educa.aragon.es/descargas/H_ Recursos/h_1_Psicol_E ducacion/h_1.2.Aspectos_sociales/1.13.Grupos_interactivos_experie ncia.pdf

Moliner, L., Amat, A. F. y Gil, J. (2010). Abriendo el aula al entorno: Transformado la comunidad universitaria mediante el Aprendizaje Servicio. Quaderns digitals: Revista de Nuevas Tecnologías y Sociedad, 10(65), 1-6.

Moliner, L., Francisco, A. y Gil, J. (2010). Abriendo el aula al entorno: Transformado la comunidad universitaria mediante el Aprendizaje Servicio. Quaderns digitals: Revista de Nuevas Tecnologías y Sociedad, 65. Recuperado de http://www. quadernsdigitals.net/index.php?accionMenu=hemeroteca.VisualizaArticulolU. visualiza\&articulo_id=10968

Píriz, R. (2011). Una experiencia de grupos interactivos en un centro de secundaria. Tendencias Pedagógicas, 17, 51-64.

Tapia, M. N. (2008). Aprendizaje y servicio solidario en la misión de la educación superior. En A. González y R. Montes (Eds.), Aprendizaje-servicio en la educación superior. Una mirada analítica desde los protagonistas (pp. 11-34). Barcelona: Eudeba.

Torío, S., Peña, J. V. y Hernández, J. (2014). Aprendizaje Servicio como entrenamiento al emprendimiento social: una experiencia universitaria. Procedia-Social and Behavioral Sciences, 139, 504-511. http://doi.org/10.1016/j.sbspro.2014.08.055

Trigueros Cervantes, C., Rivera García, E., Moreno Doña, A. y Muñoz Luna, R. (2016). Uso del software CAQDAS Nvivo en Ciencias Sociales para la investigación con grupos de discusión. Index de Enfermería, 25(3), 171-174.

Trigueros, C., Rivera, E. y Rivera, I. (2019). Técnicas conversacionales y narrativas Investigación cualitativa con Software NVivo. Granada: UGR y EASP. 
Trigueros, C., Rivera, E. y Rivera, I. (2017). The Use of NVivo in the Different Stages of Qualitative Research. En A. P. Costa, L. P. Reis, F. N. de Souza y A. Moreira (Eds.), Computer Supported Qualitative Research: Second International Symposium on Qualitative Research (ISQR 2017) (pp. 381-392). Cham: Springer International Publishing. https://doi.org/10.1007/978-3-319-61121-1_32

Wilczenski, F. L. y Cook, A. M. (2009). How service learning adresses the mental health needs of students in urban schools. Journal of School Counseling, 7(25), $1-20$. 